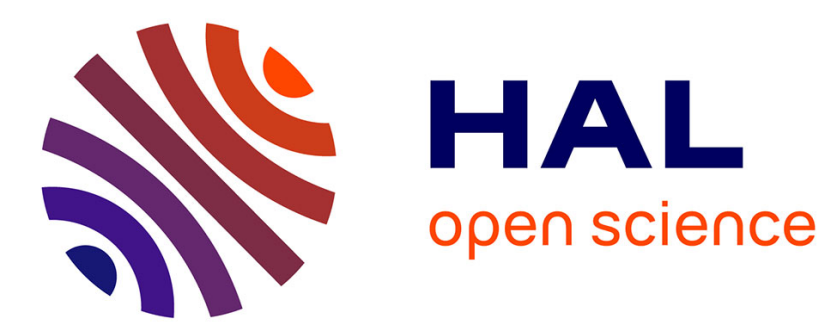

\title{
Quince (Cydonia oblonga Mill.)
}

Michel Duron, Luc Decourtye, Philippe Druart

\section{To cite this version:}

Michel Duron, Luc Decourtye, Philippe Druart. Quince (Cydonia oblonga Mill.). Trees II, 5, SpringerVerlag, 1989, Biotechnology in Agriculture and Forestry, 3540191585. hal-02857966

\section{HAL Id: hal-02857966 https: / hal.inrae.fr/hal-02857966}

Submitted on 8 Jun 2020

HAL is a multi-disciplinary open access archive for the deposit and dissemination of scientific research documents, whether they are published or not. The documents may come from teaching and research institutions in France or abroad, or from public or private research centers.
L'archive ouverte pluridisciplinaire HAL, est destinée au dépôt et à la diffusion de documents scientifiques de niveau recherche, publiés ou non, émanant des établissements d'enseignement et de recherche français ou étrangers, des laboratoires publics ou privés. 


\section{I.4 Quince (Cydonia oblonga Mill.)}

M. Duron, L. Decourtye ${ }^{1}$, and Ph. Druart ${ }^{2}$

\section{Introduction}

\subsection{Importance of the Plant}

Quince (Cydonia oblonga Mill.) belongs to the Rosaceae family and is the only species in the genus. It derives its generic name from Cydon, the modern Canea, near which the tree grew in great abundance (Evreinoff 1948). It is a deciduous unarmed shrub or small tree, which usually attains a height of less than 15 feet (Fig. 1). Differing from other pip fruits, blooming is terminal and solitary at the extremity of current-season growth. Leaves are densely pubescent below, dull green above. Fruits have a woolly appearance and are fragrant near maturity. Each ovary contains five compartments, each of them with numerous ovules, which produce seeds with a sticky jelly. Trees are partly self-pollinated, but fruit setting and seed number per fruit are increased by cross-pollination. According to fruit shape, different types are usually distinguished:

C. o. pyriformis (Kirchn.) Rehd. var., which is the typical form, pear-shaped, without ribs.

C. o. maliformis (Mill.) Schneid. var., with roundish fruit, is more like an apple. C. o. lusitanica (Mill.) Schneid. var., which is also pear-shaped, but obviously ribbed.

Flesh is firm, yellow, weakly juicy and of low palatability for eating raw. The uses of the quince fruit are limited mostly to the making of jelly, marmalades and preservee, or for adding flavour to apples and pears when stewed or baked. Besides its limited production for fruiting, quince is widely used as a pear rootstock for its dwarfing effect. Quince roots produce a tree $30 \%$ to $60 \%$ of size on Pyrus seedlings, and, in most conditions, hasten the time of fruiting. Most of European pear orchards and some American ones, are grown on quince. Although this graft compatibility between two different genera is still quite uncommon, quince can also be used for other fruit or ornamental genera like Mespilus, Sorbus, Amelanchier, Eryobothrium, Crataegus, Raphiolepis etc.

This wide flexibility for other genus relationships has also to be compared with the rare phenomenon of graft hybrids, obtained at the beginning of the 20th

${ }^{1}$ I.N.R.A. Fruit Breeding Station, Beaucouzé, 49000 Angers, France

2 Agronomy Research Centre, Research Station, 234 Chaussée de Charleroi, 5800 Gembloux, Belgium

Biotechnology in Agriculture and Forestry, Vol. 5

Trees II (ed. by Y.P.S. Bajaj)

(c) Springer-Verlag Berlin Heidelberg 1989 
Fig. 1. Six-year-old quince cv. Portugal at the INRA's Fruit Breeding Station in Angers (1976)

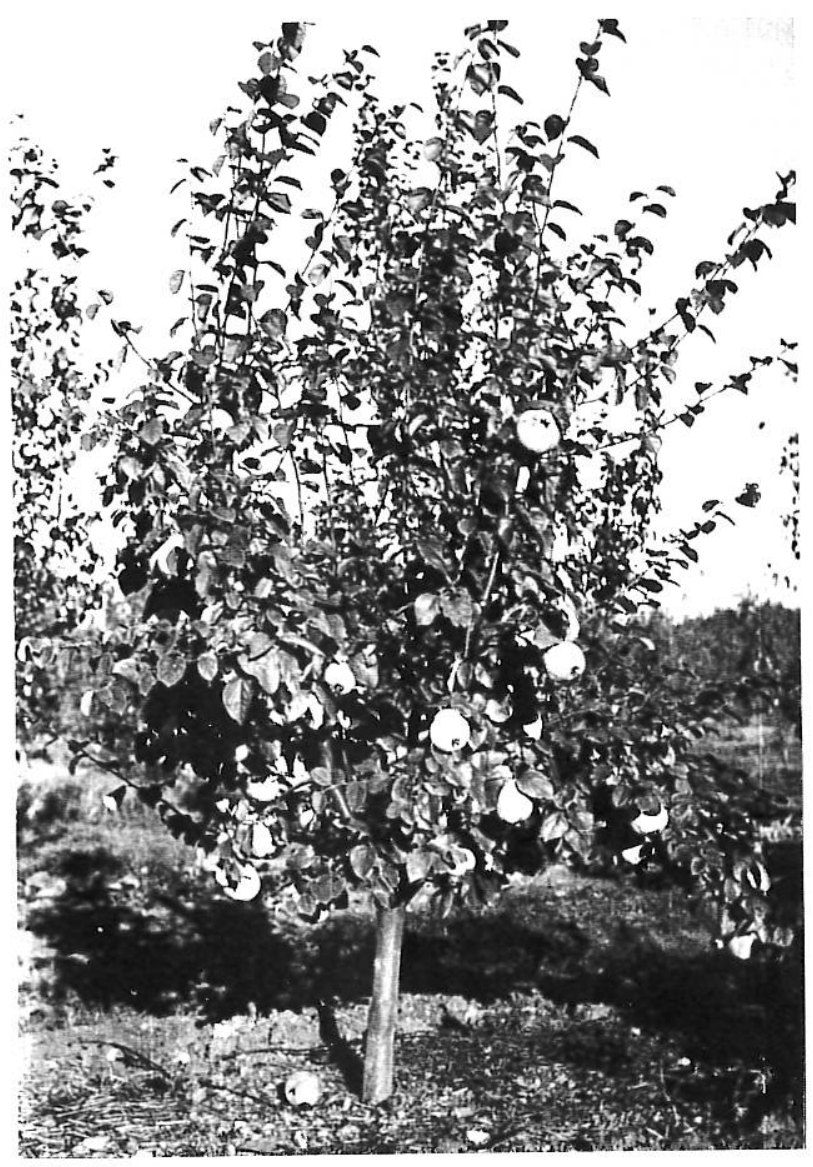

century, + Pyrocydonia Danieli (Winkl.) Rehd., and + Pyrocydonia Winkleri (Daniel) Rehd. Sexual hybrids with pears, Pyronia have also been obtained at different times: P veitchii (Trabut) Guillaumin, originated before 1913, and has since been obtained in England and the Soviet Union (Rudenko 1978). Sexual hybrids with apple (Malus pumila) have also been reported.

Much selection work has been carried out in Europe in this century, starting in England near 1930, to a lesser extent in Germany, Poland, Belgium, and, more recently (1950-1980), in France (Brossier 1965).

\subsection{Distribution and Area Under Cultivation}

The quince is a native of Western Asia, from Iran (Persia) to Turkestan. Its use seems to be very old, starting from Persia some 4000 years B.C. and spreading with the prosperous civilisations of that time all over the Mediterranean basin to the west, and to Afghanistan to the east.

Nowadays, quince fruit production is prevalent in the Balkans, Turkey, Hungary, Italy, France, Spain, Morocco, but mainly in Iran, Caucase, Turkestan 


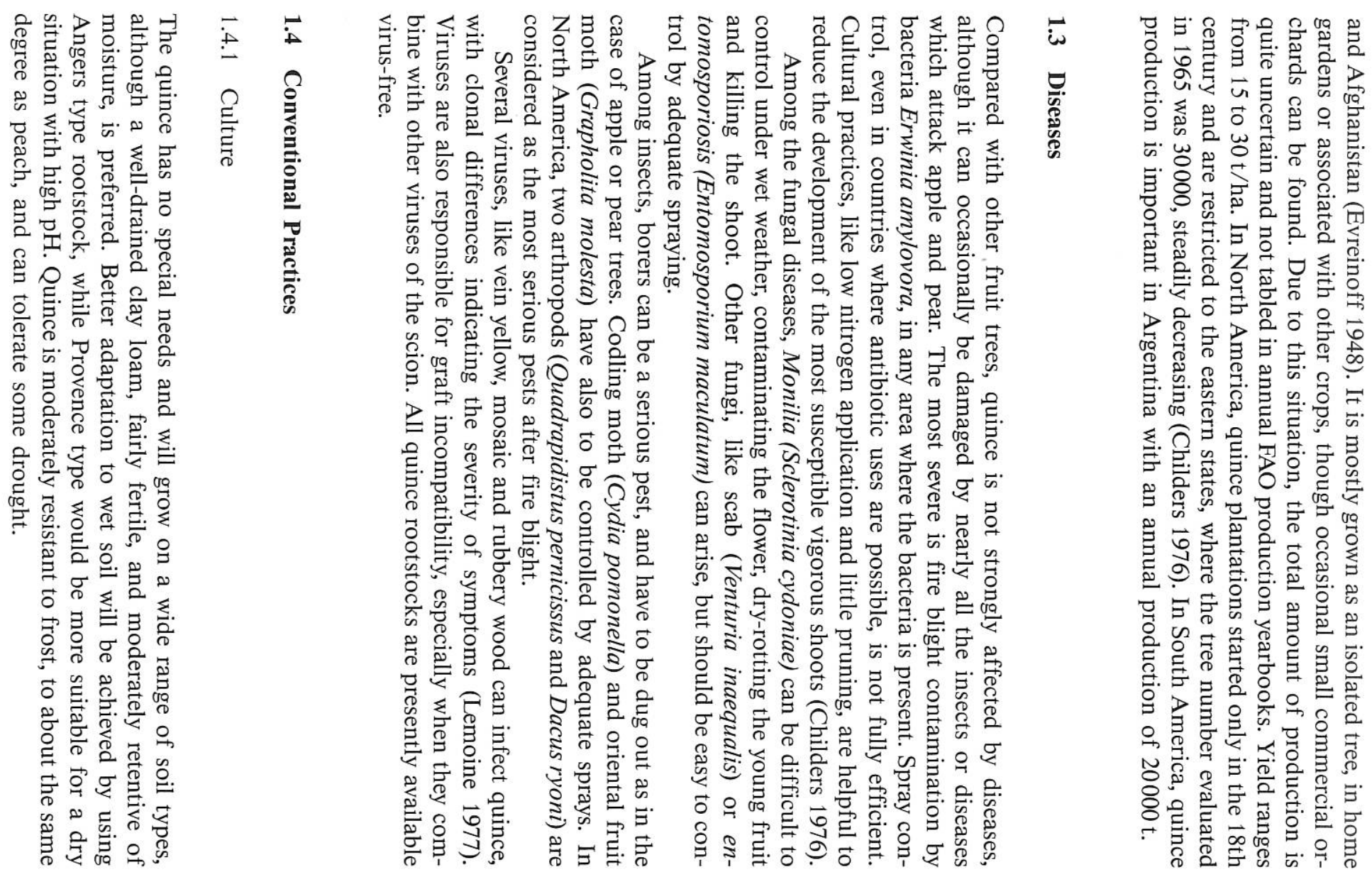

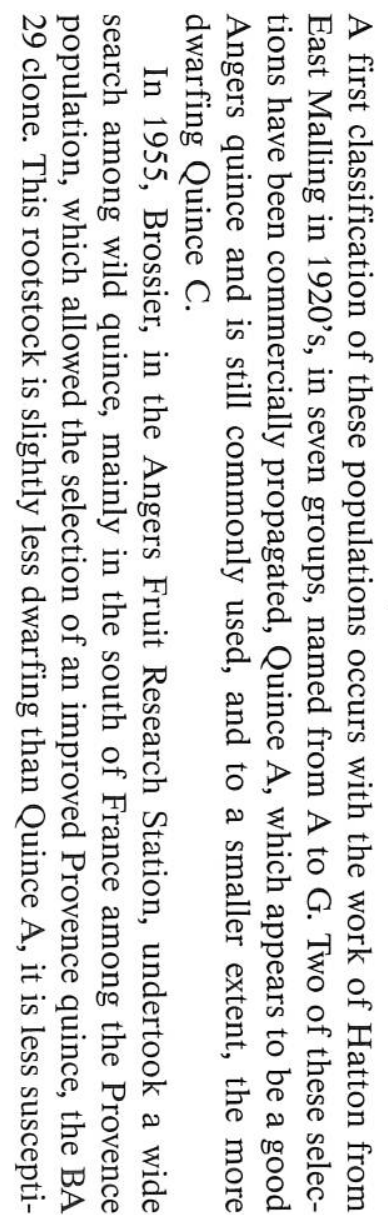

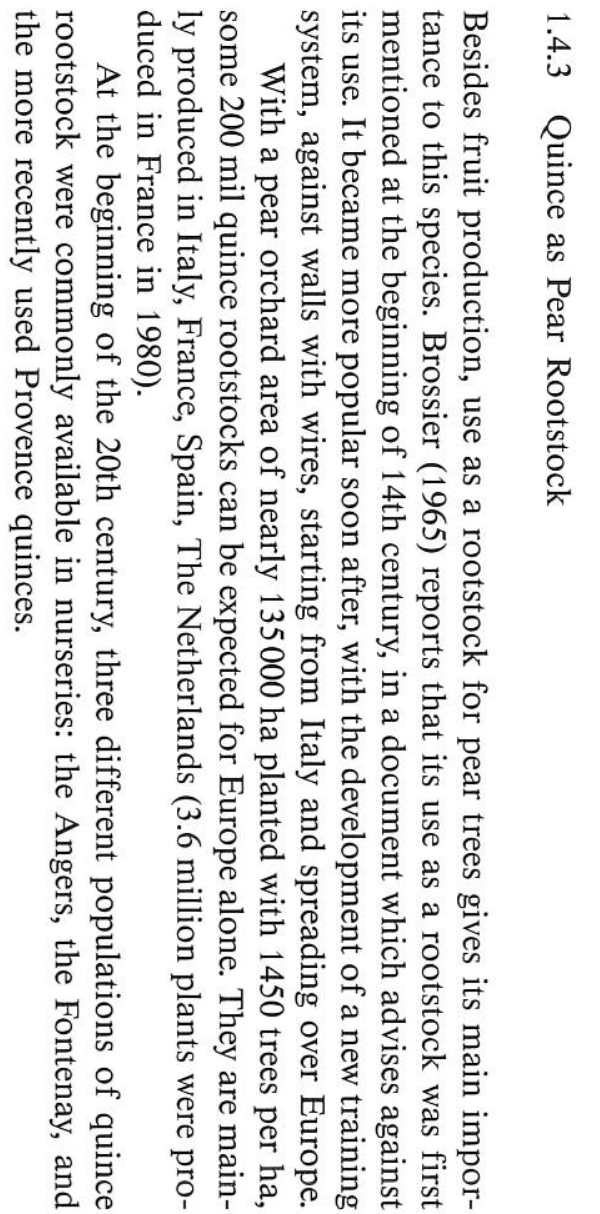

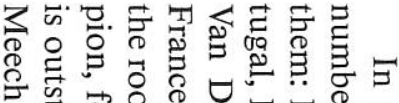

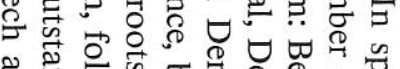

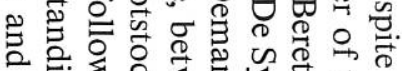

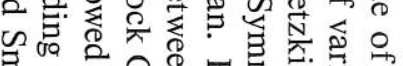

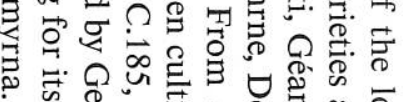
के

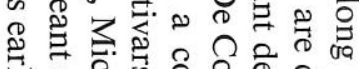
ज्ञे के की

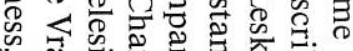

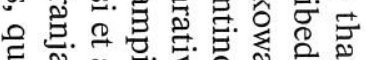

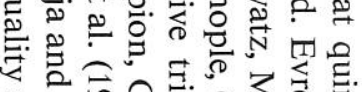

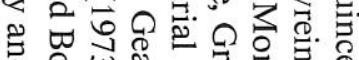

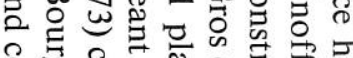

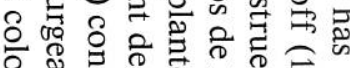
导芑

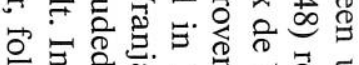

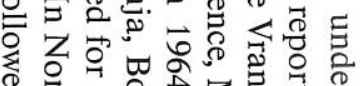

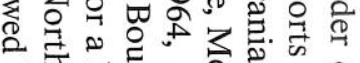
व

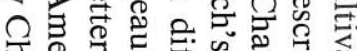

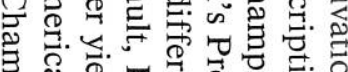

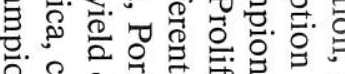

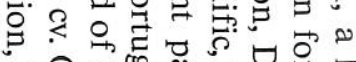

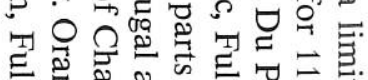

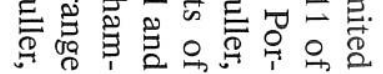



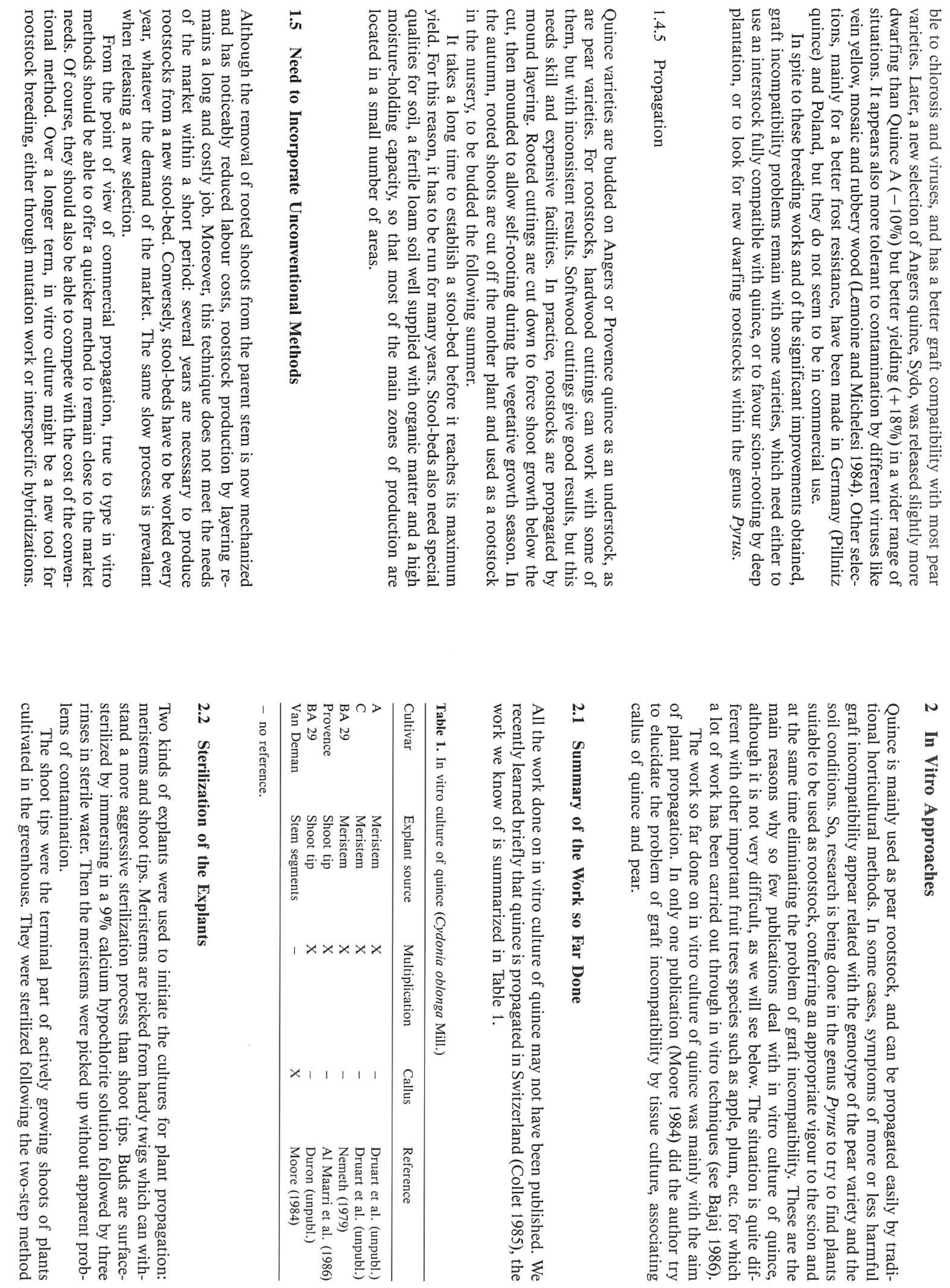

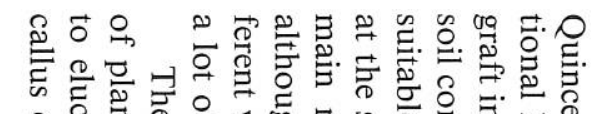

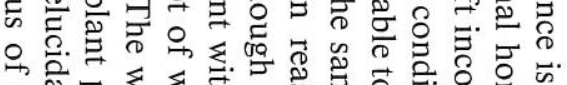

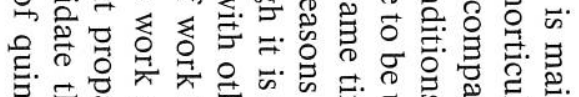

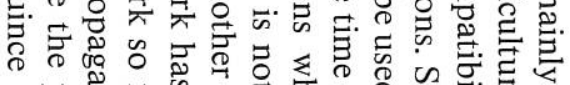

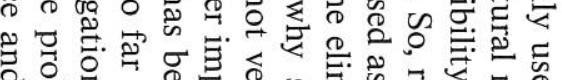

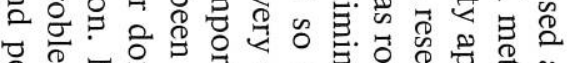

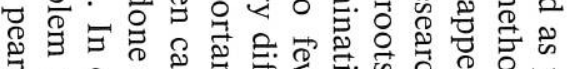

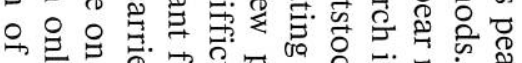

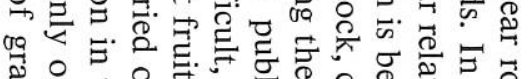

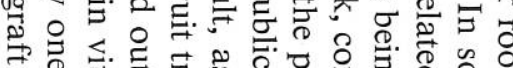

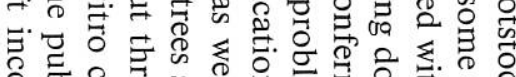

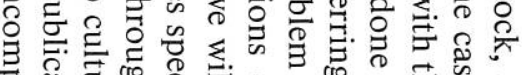

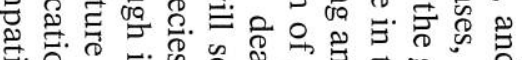

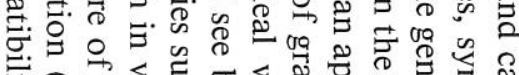

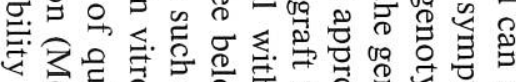

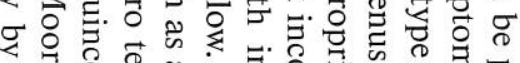

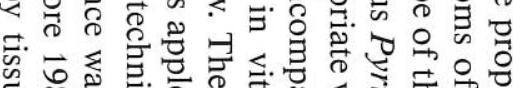

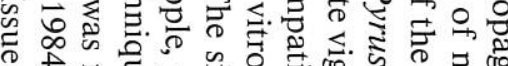
き.

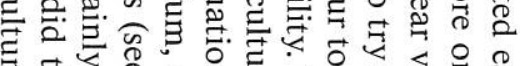

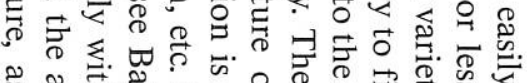
卷芯氮.

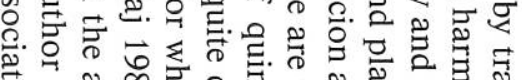

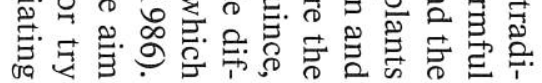




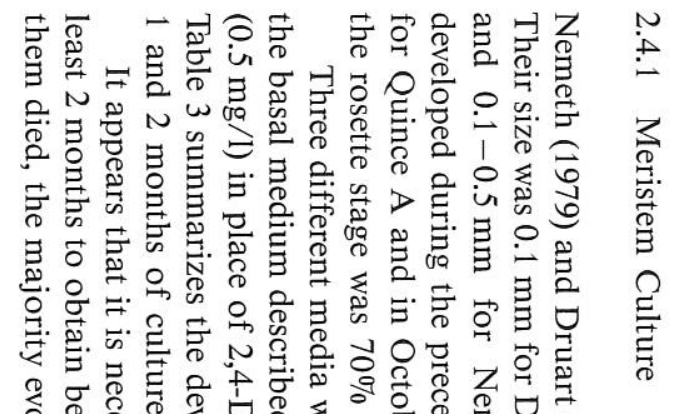

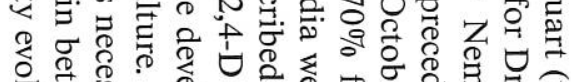

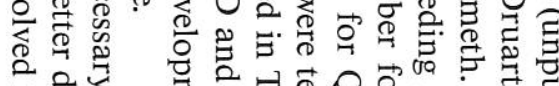

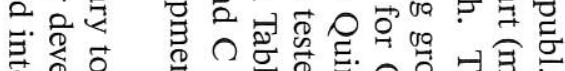

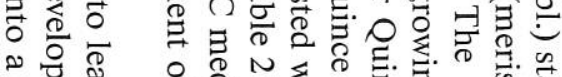

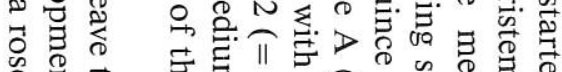

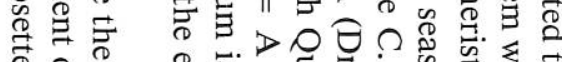

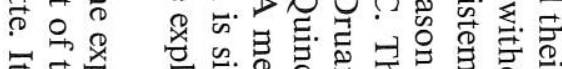

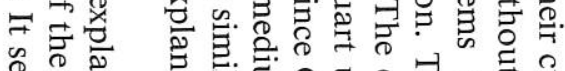

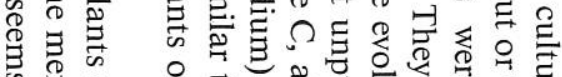

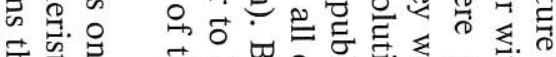

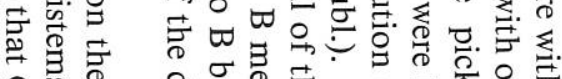

ด

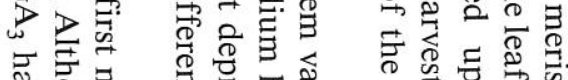

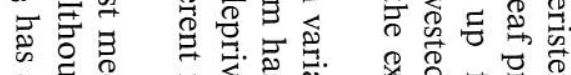

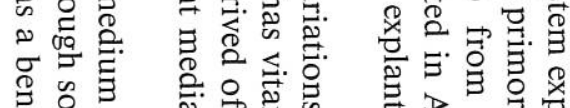

营 寻

ํํㄹㅇㅠ

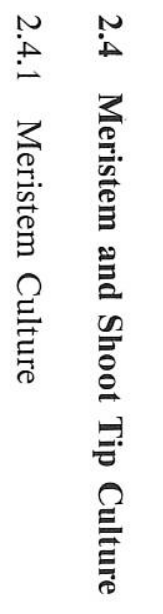

ڤี

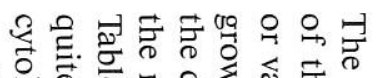

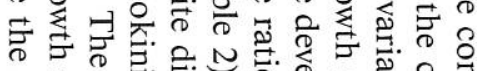

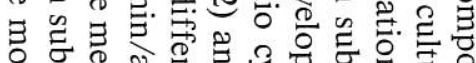

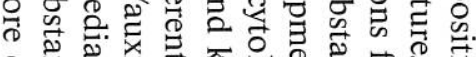

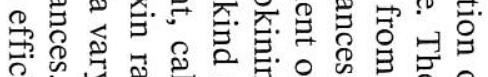

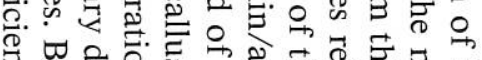

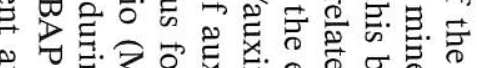

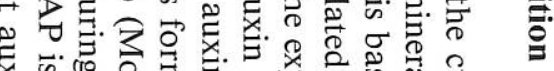

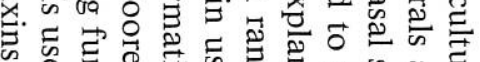

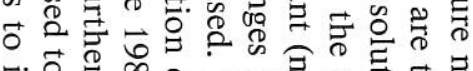

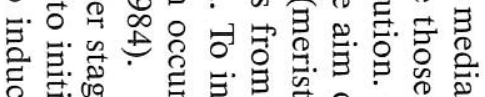

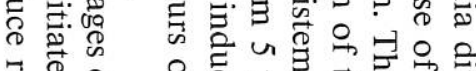

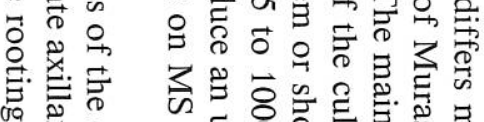

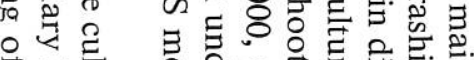

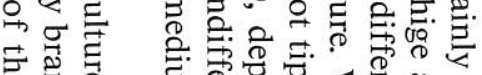

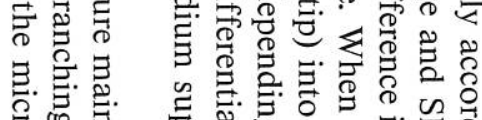

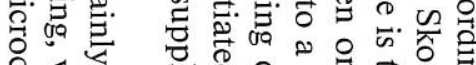

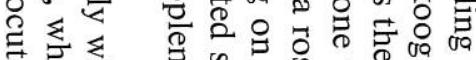

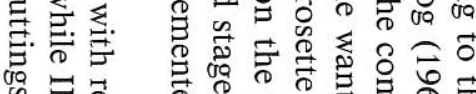

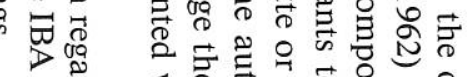

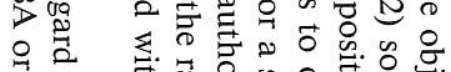

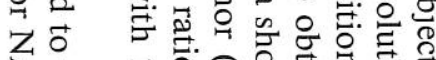

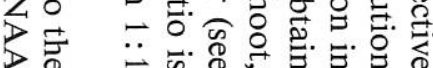

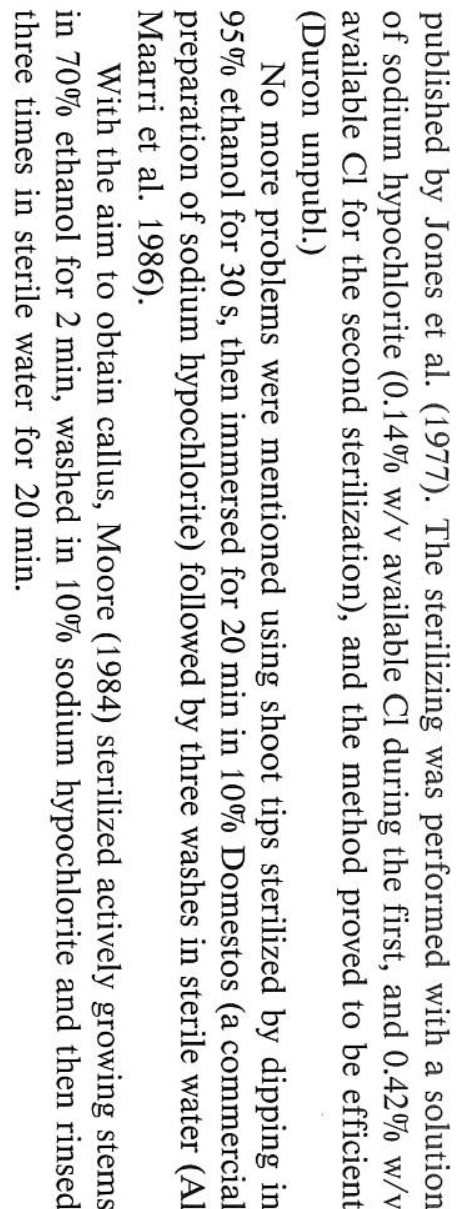

Table 2. Meristem, shoot tip and stem segments culture media

\begin{tabular}{|c|c|c|c|c|c|}
\hline \multirow{2}{*}{$\begin{array}{l}\text { Explants } \\
\text { Reference }\end{array}$} & \multicolumn{2}{|l|}{ Meristems } & \multicolumn{2}{|l|}{ Shoot tips } & \multirow{2}{*}{$\begin{array}{l}\text { Stem segments } \\
\text { Moore (1984) }\end{array}$} \\
\hline & Druart (1980) & Nemeth (1979) & Al Maarri et al. (1986) & Duron & \\
\hline Macronutrients & $\mathrm{MS} / 2$ & Walkey's medium & Lepoivre (1978) & MS & MS \\
\hline Micronutrients & Nitsch & & Lepoivre $(1978)^{a}$ & MS & MS \\
\hline Vitamins (mg/l) & Jacquiot (Gautheret 1959) & Boxus and Quoirin (1974) & & & \\
\hline Myoinositol & & & 100 & 100 & MS \\
\hline Thiamine, $\mathrm{HCl}$ & & & 0.4 & 0.4 & \\
\hline \multicolumn{6}{|c|}{ Growth regulators (mg/l) } \\
\hline Kin & - & Boxus and Quoirin (1974) & - & - & 0.2 \\
\hline BAP & 1 & & 0.5 & 1 & \\
\hline $\mathrm{GA}_{3}$ & 0.1 & & 0.2 & 0.5 & - \\
\hline IBA & - & & 0.1 & 0.1 & - \\
\hline $2,4-\mathrm{D}$ & 0.001 & & - & - & 0.25 \\
\hline Sucrose $(g / 1)$ & 20 & 30 & 30 & 30 & 30 \\
\hline Agar $(g / 1)$ & 6 & 6 & 6 & 7 & NS \\
\hline $\mathrm{pH}$ & 5.8 & NS & $5.5-5.7$ & 5.7 & NS \\
\hline
\end{tabular}

NS $=$ not specified.

${ }^{\text {a }} \mathrm{Fe}$ added in Al Maarri's media as Fe $\mathrm{Na}$ EDTA $=20 \mathrm{mg} / \mathrm{l}$. 


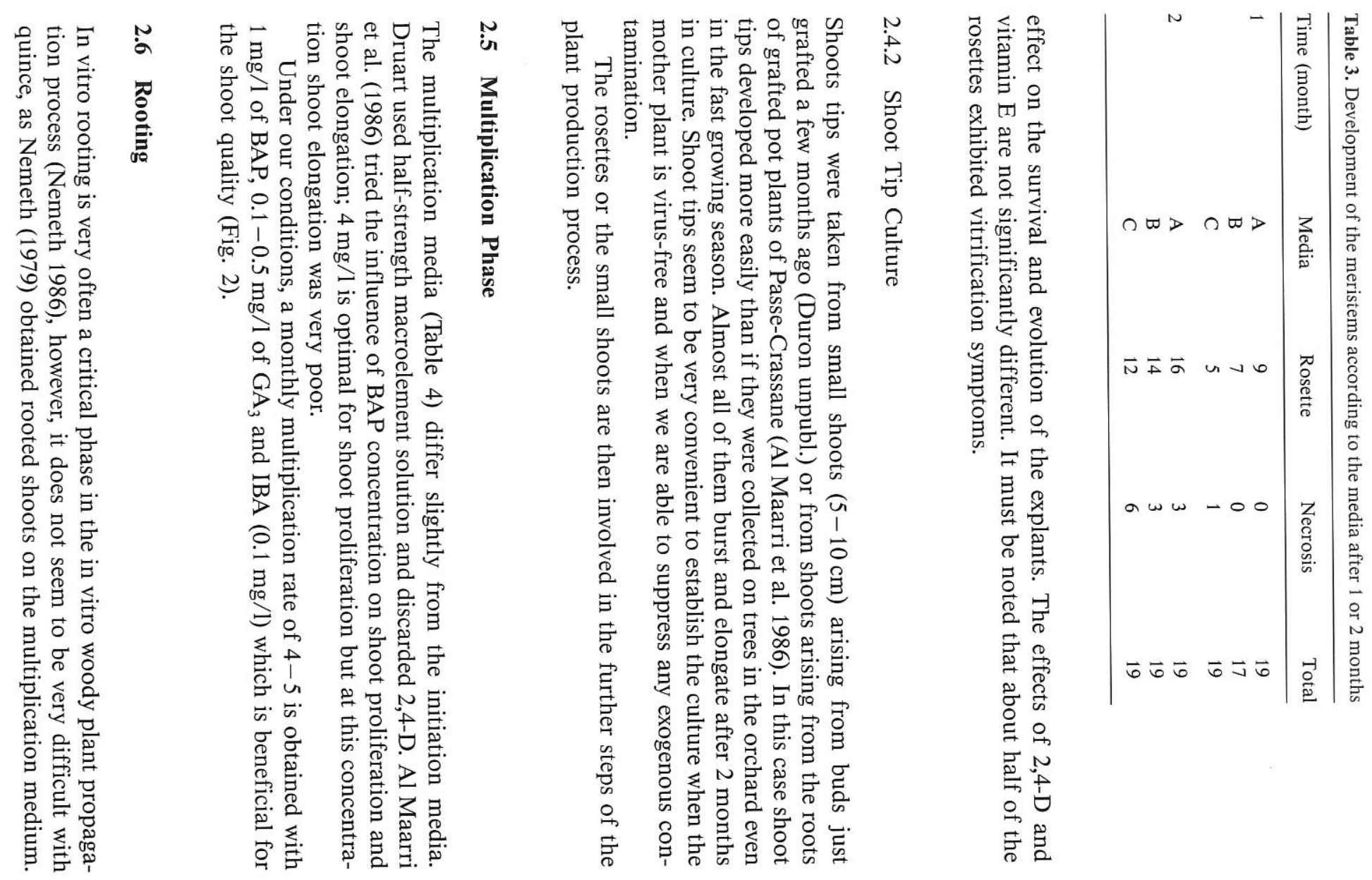

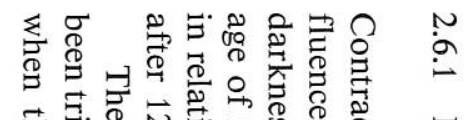

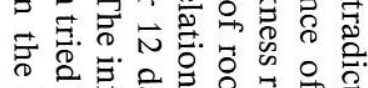

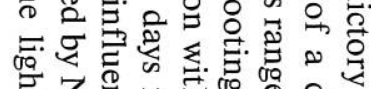

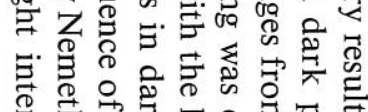

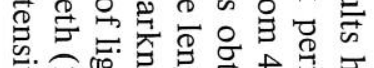

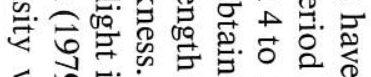
«

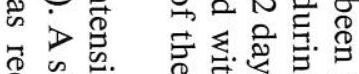

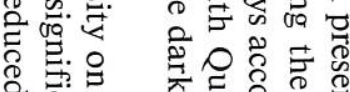

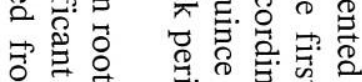

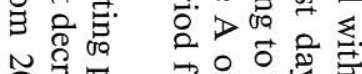
슝 范

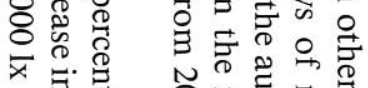
岀司莒 व융 कि

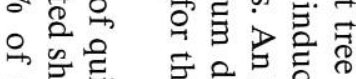

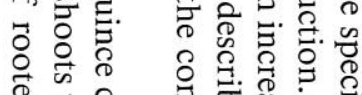
造?

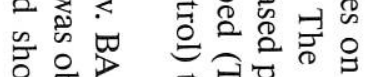

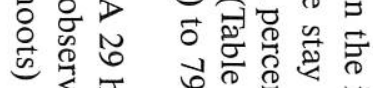

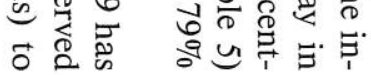

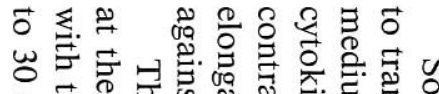

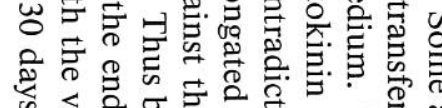

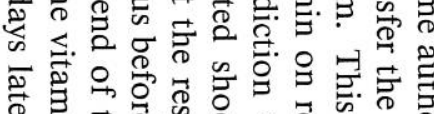

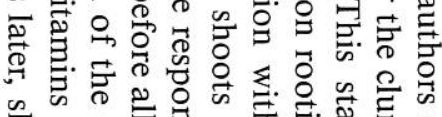

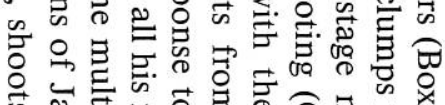

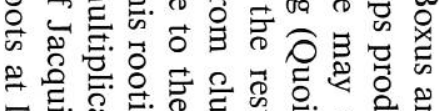

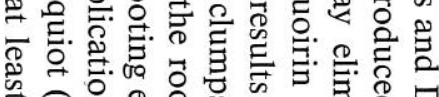

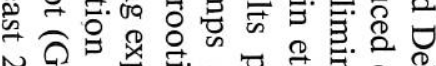

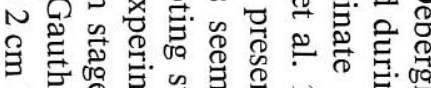

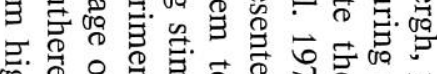

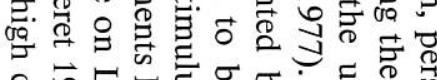

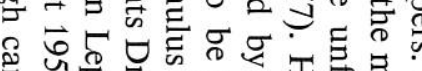

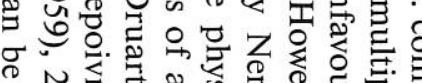

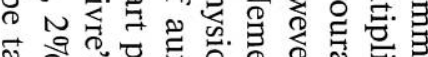

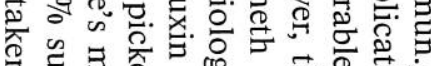
क

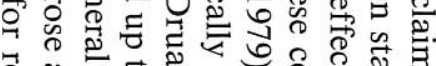

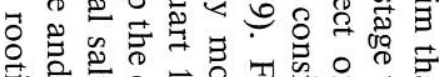

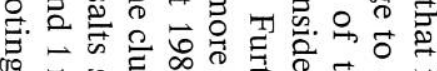

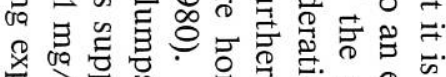

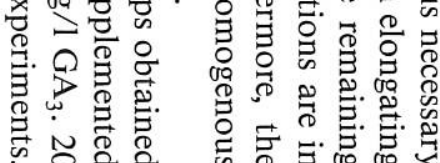

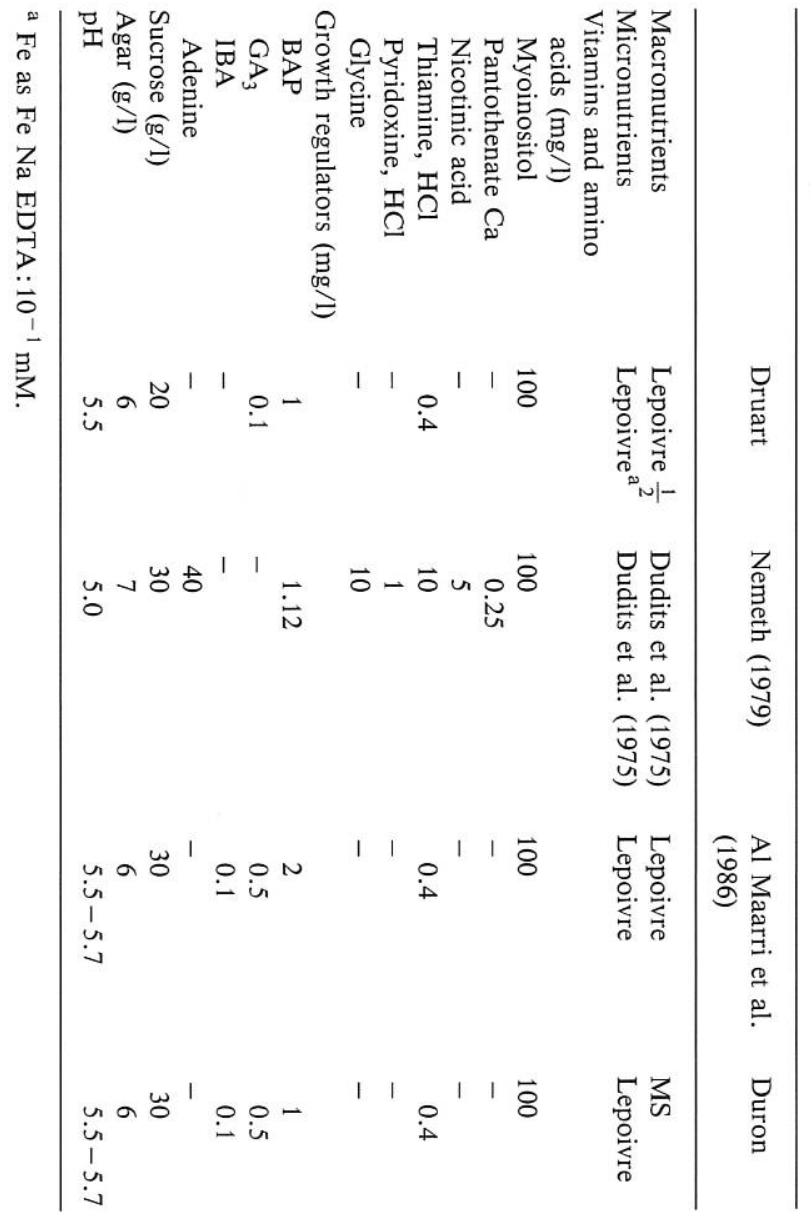




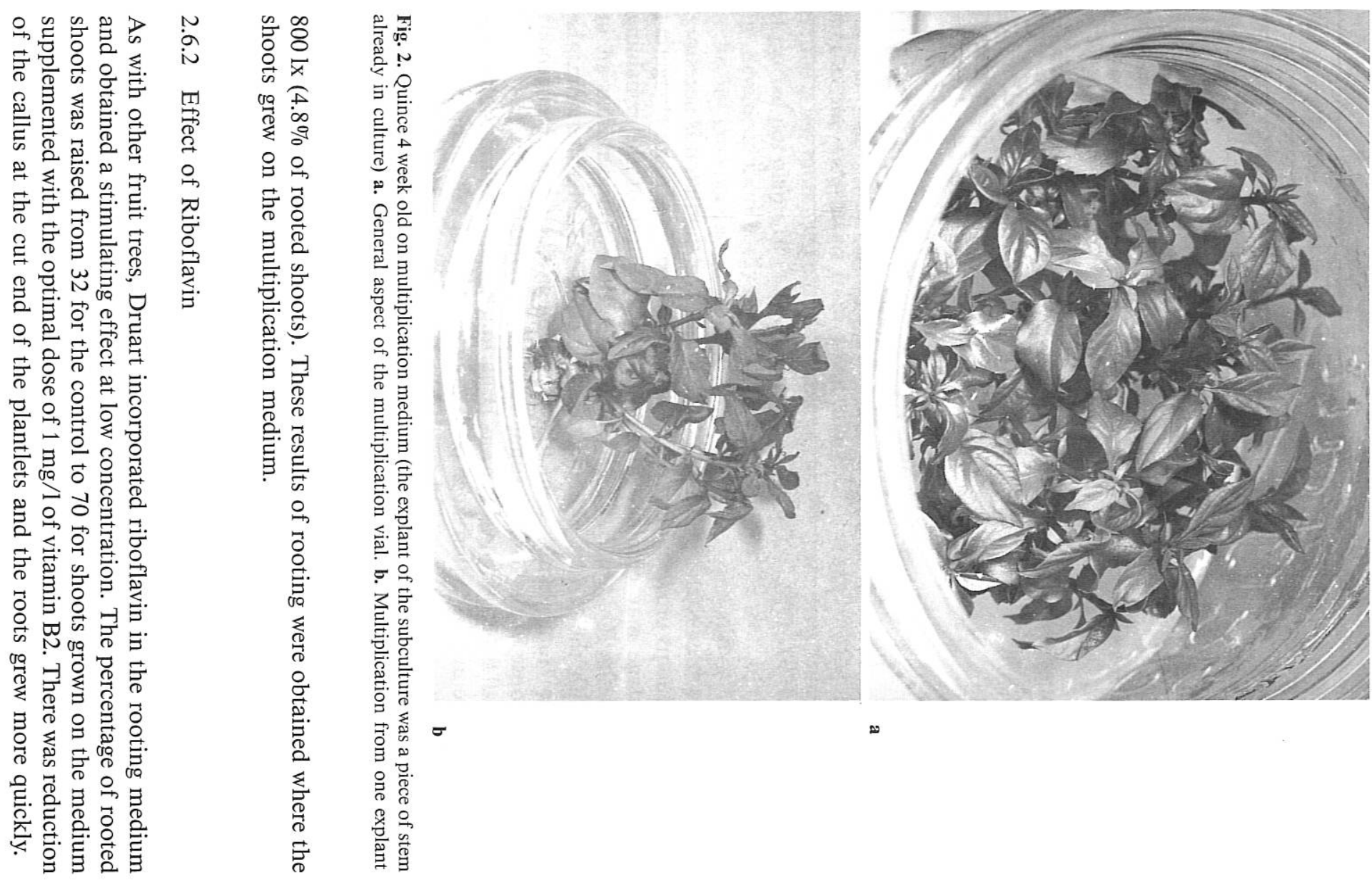

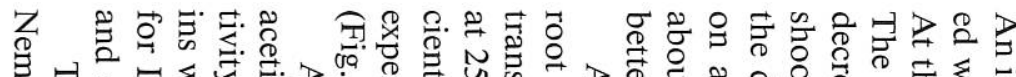

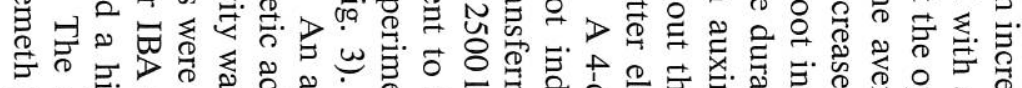

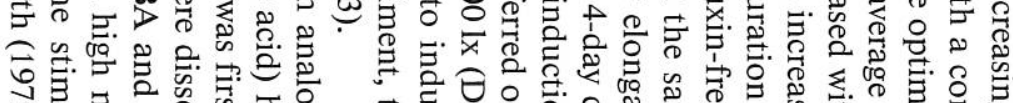

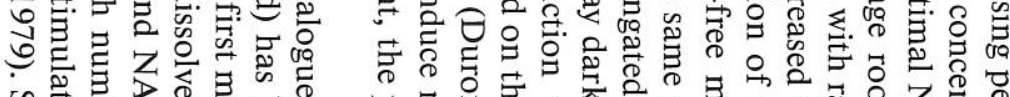

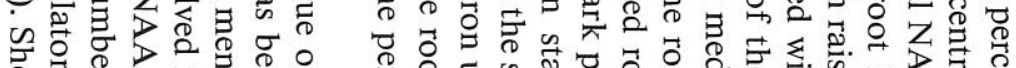

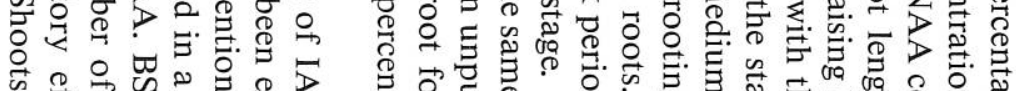

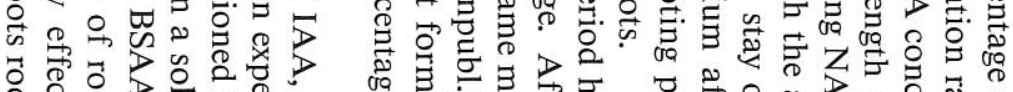

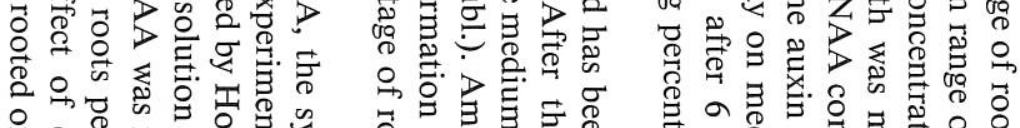

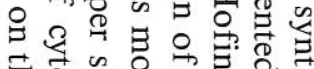

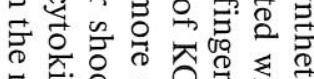

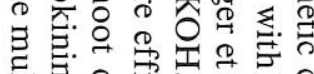

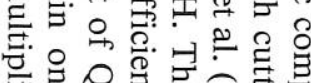

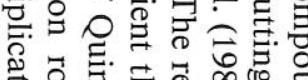

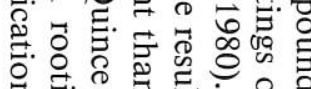

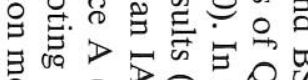

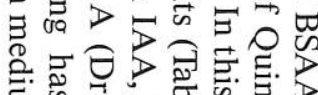

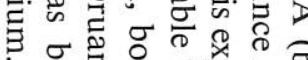

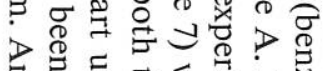

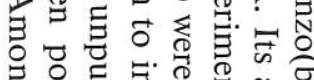

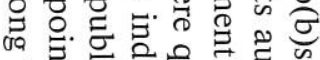

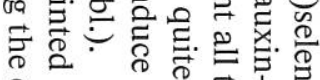

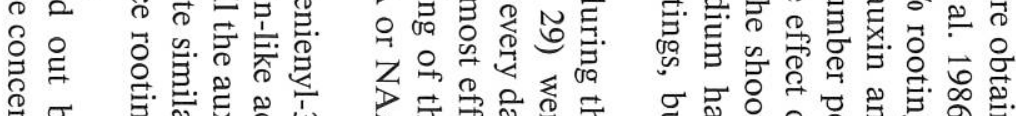

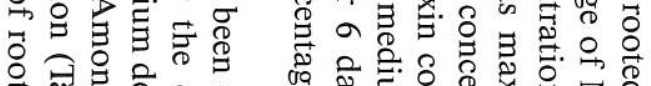

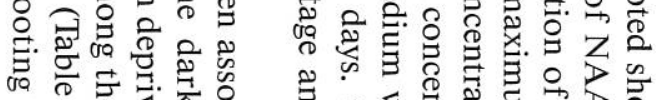

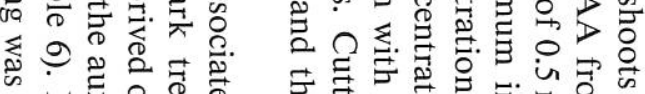

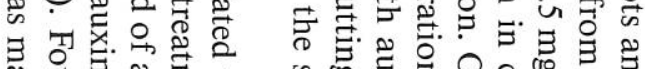

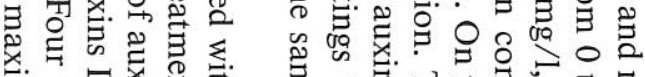

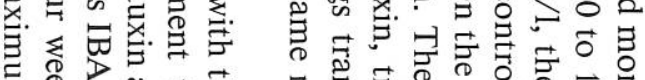

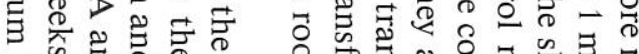

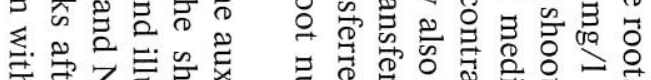

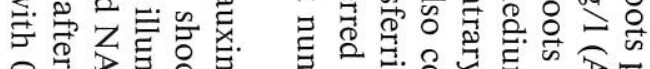

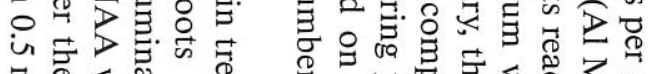

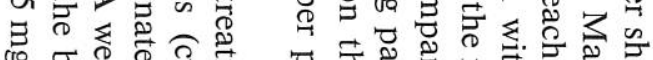

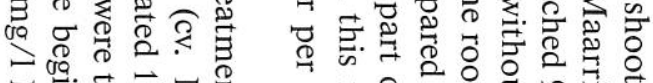

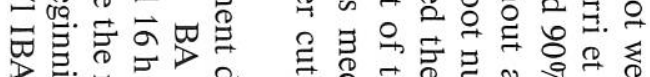

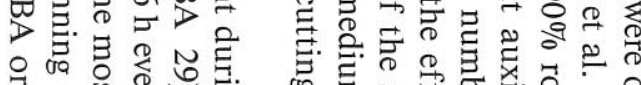

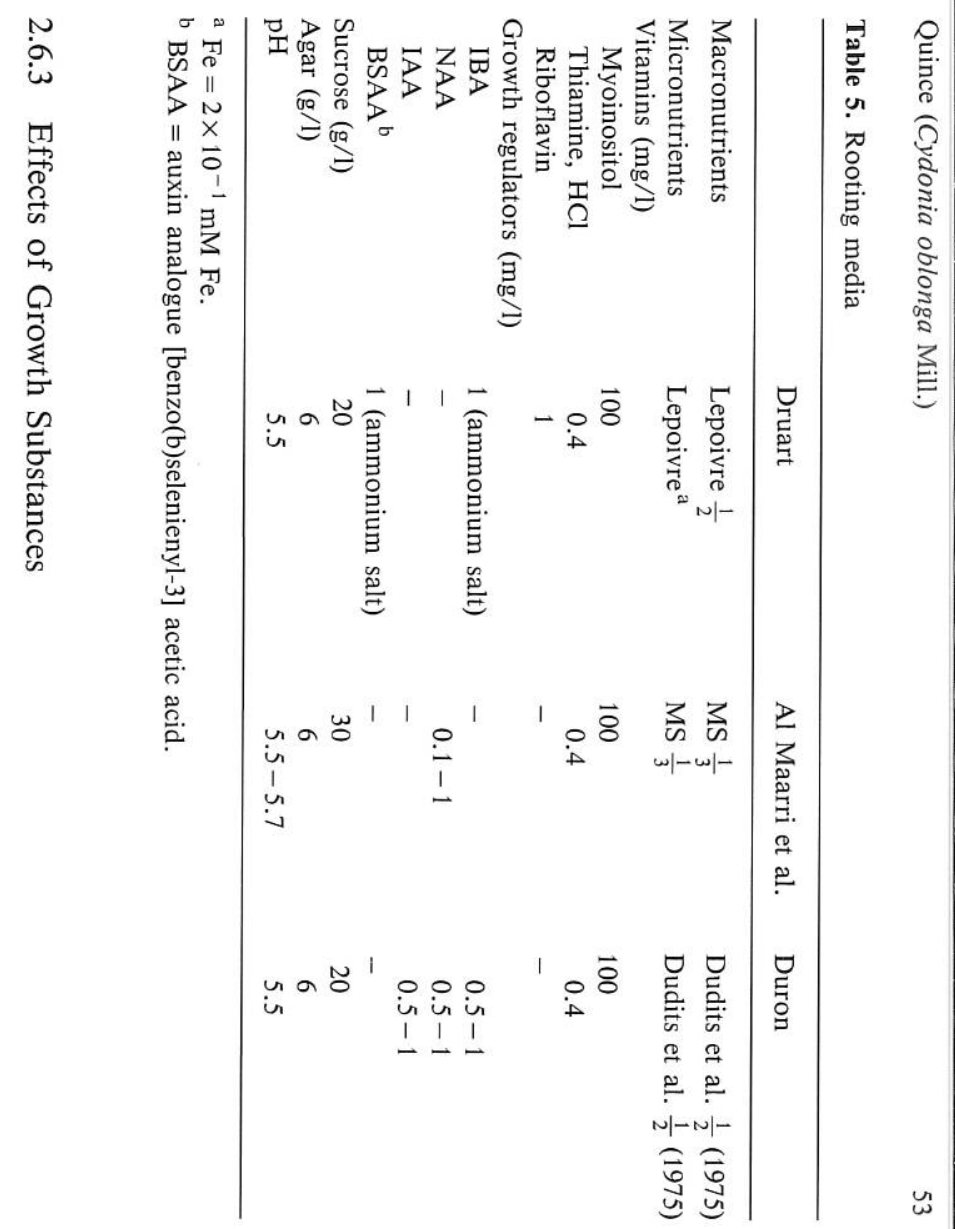



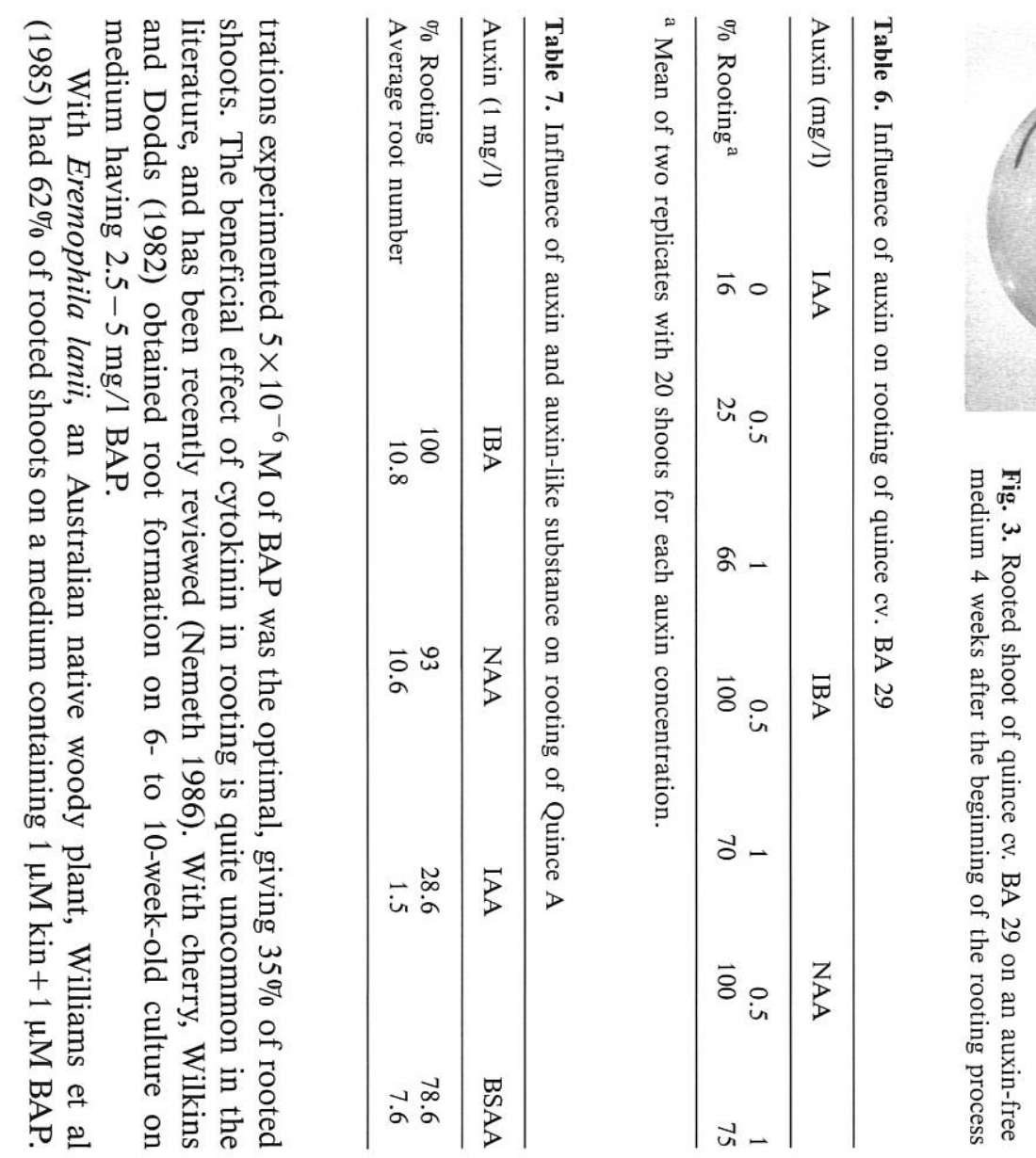

us

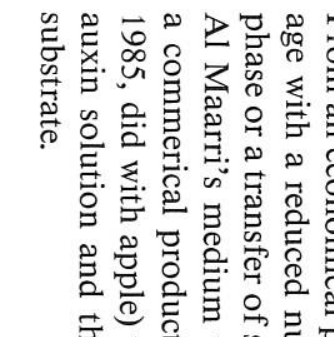

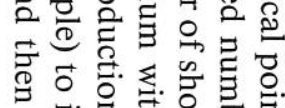

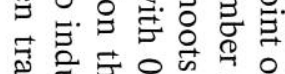

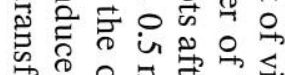

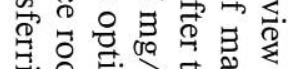

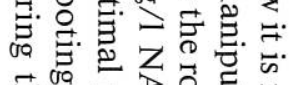

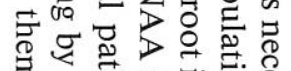

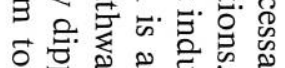

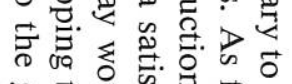

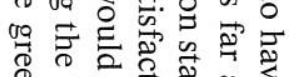

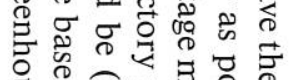

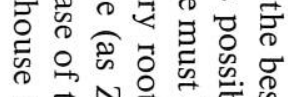

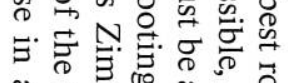

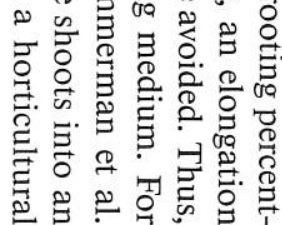

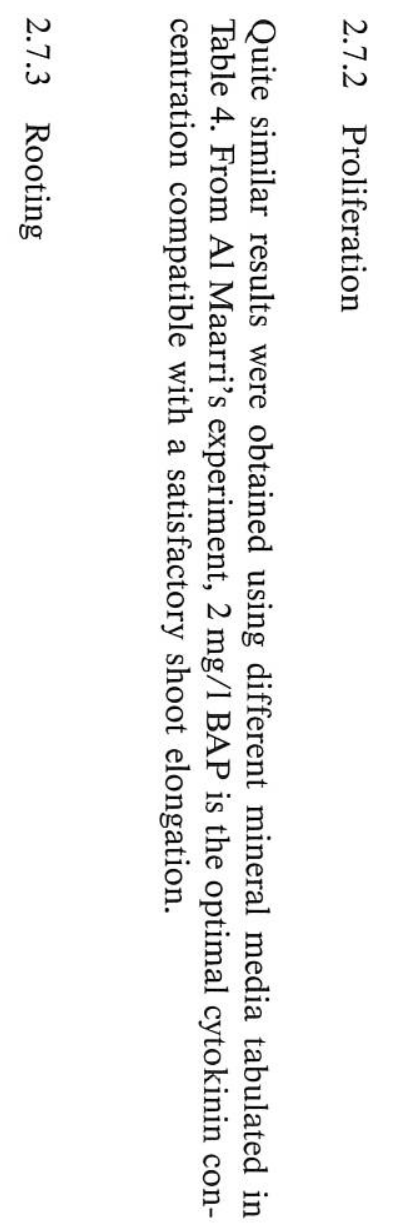

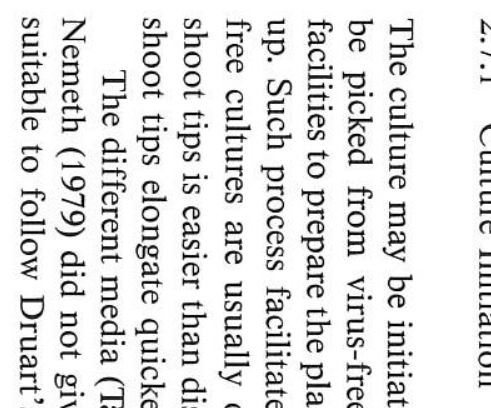

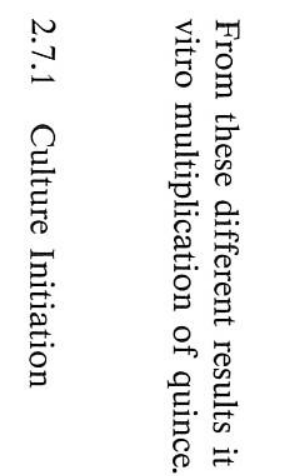

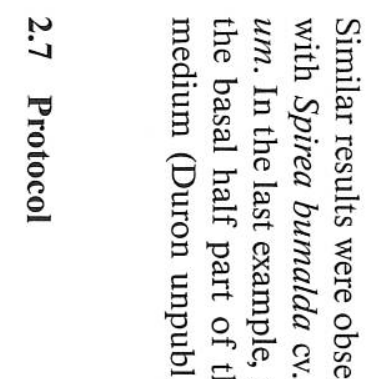

क ठิ

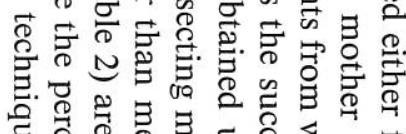

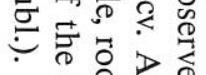

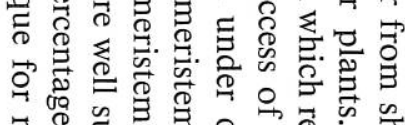

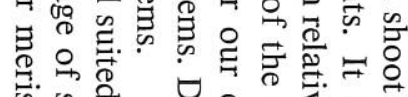

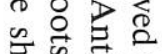

ठํㅇㅇㅛ

के

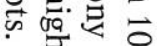

$\exists$ 声婇

声节兽交

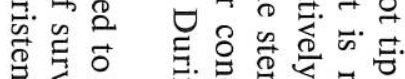

‡

s.

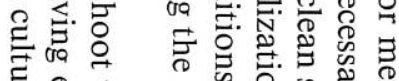

急号施

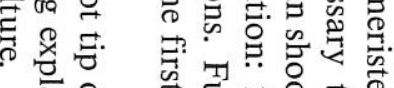

용.

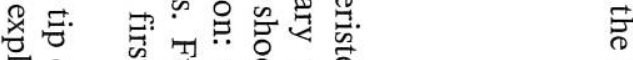

के

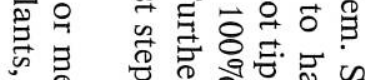

官官

管点.

寻 䆑.

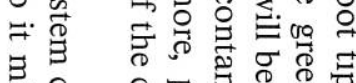

는

罗.

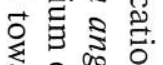

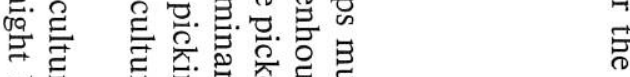

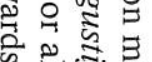

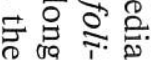




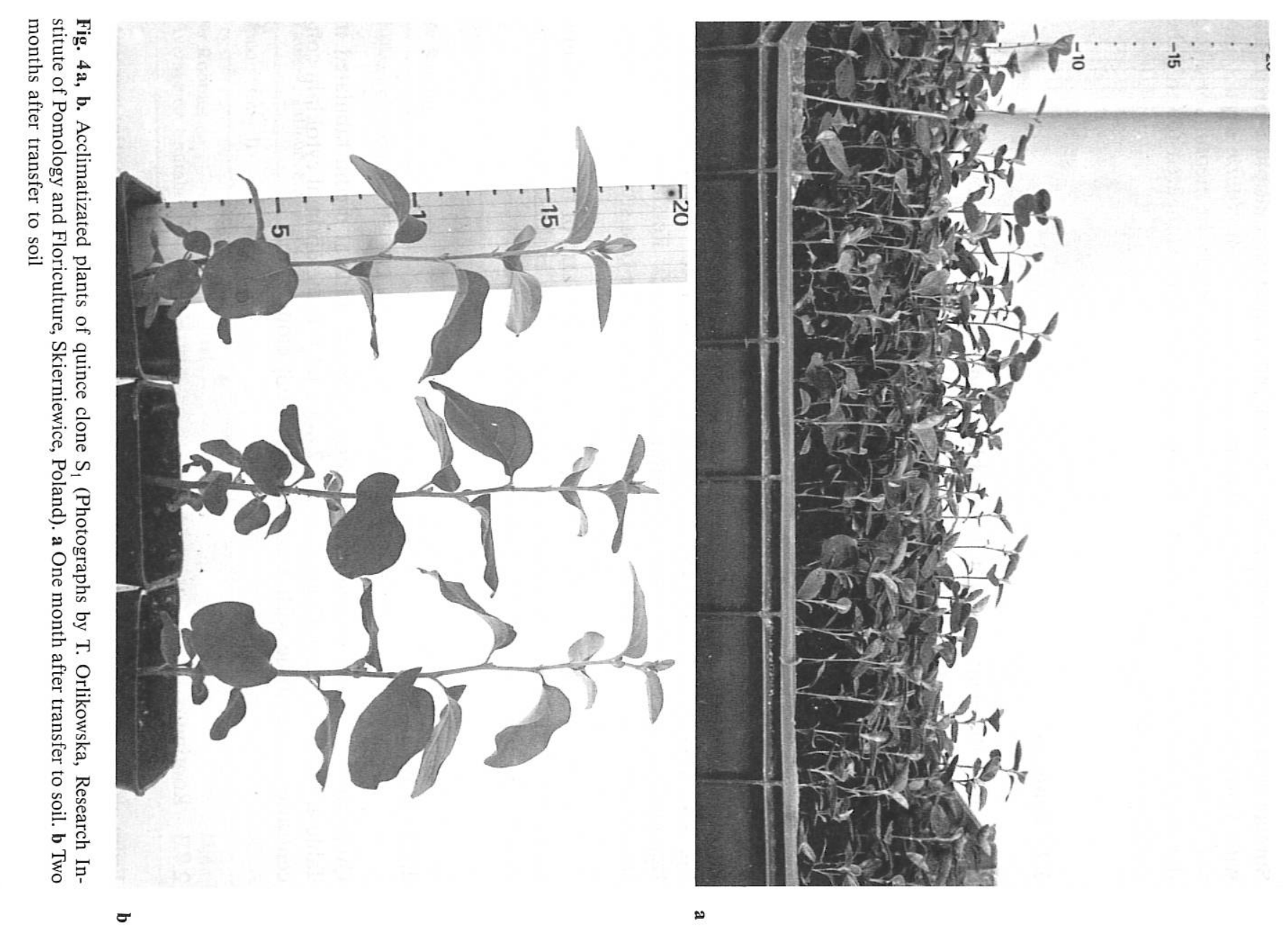

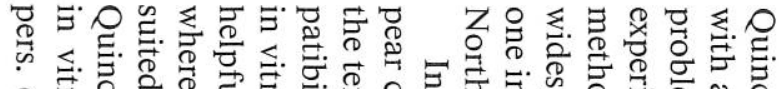
ภ

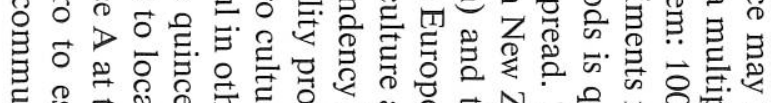

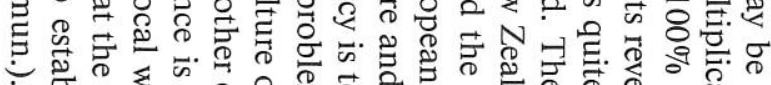

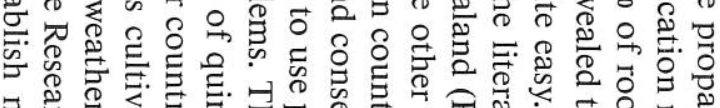

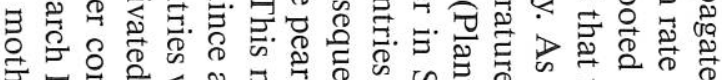

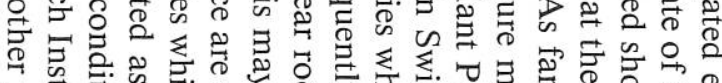

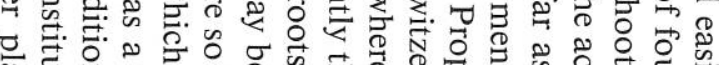
害㐘

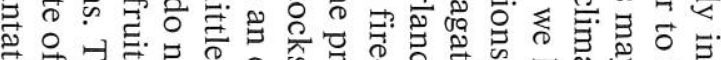

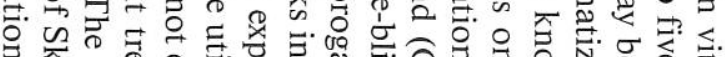

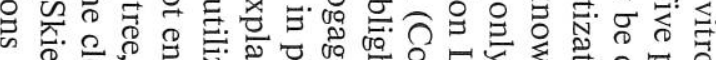

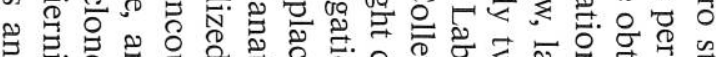

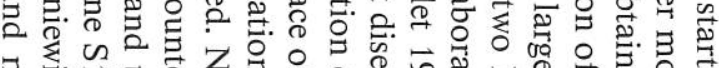

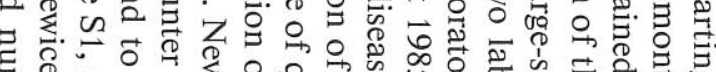

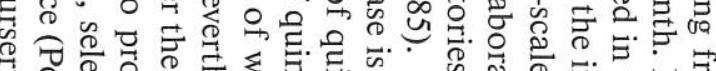

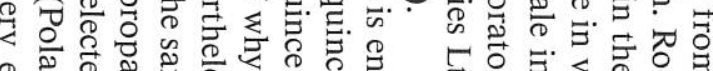

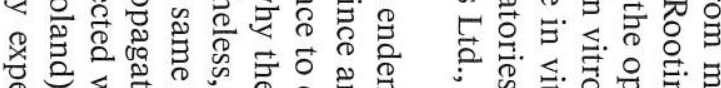

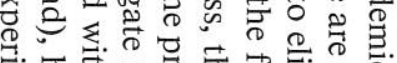

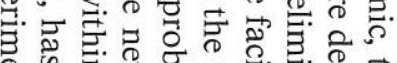

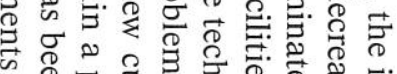

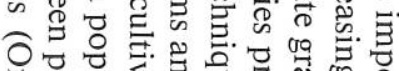

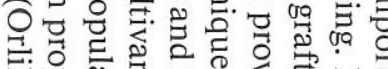

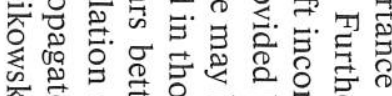

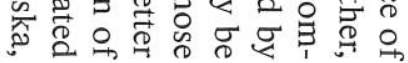

ช ○ 동

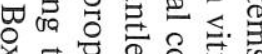

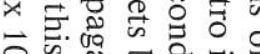
○ क

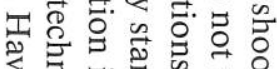

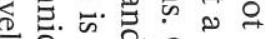

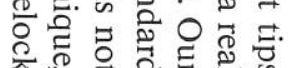

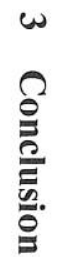

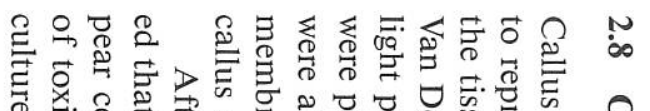

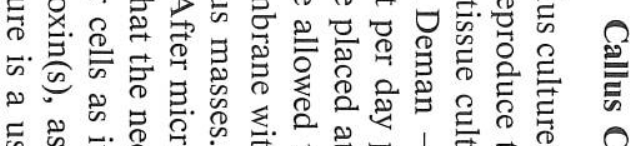

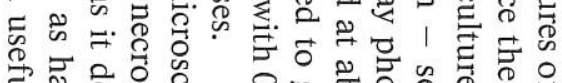
总节命

त्व

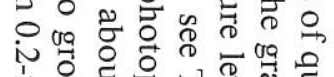

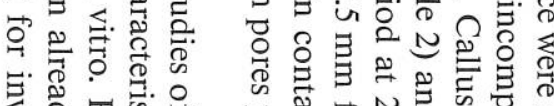

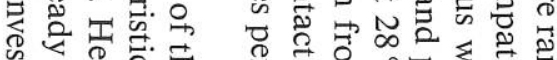

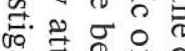

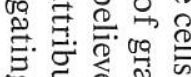

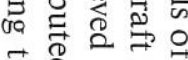

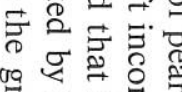
里葛葛

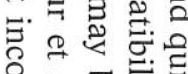

Ð

范䓂

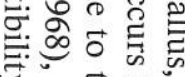
它䆑寻

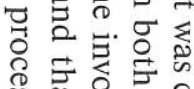

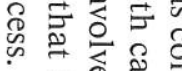

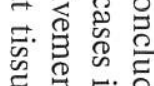
寻寻

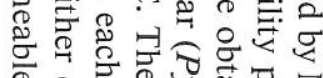

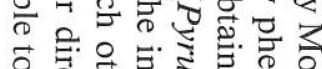

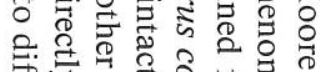

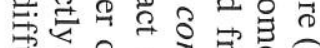

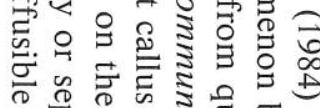

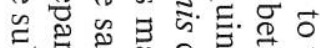

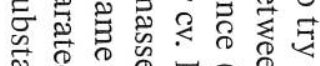
Ð ฉ

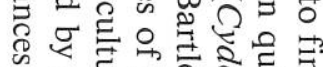
क छ

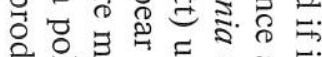

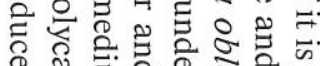

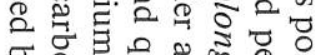

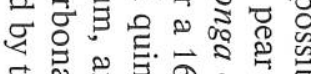

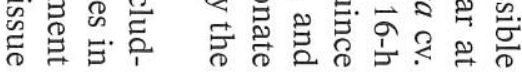

웅 ซ

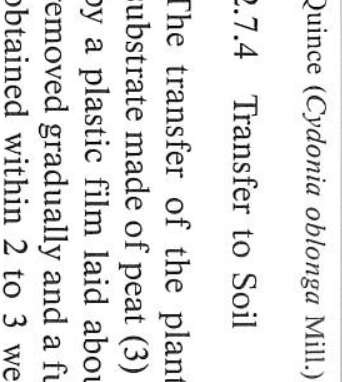

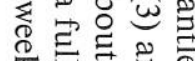
不光苛票

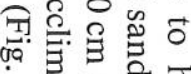

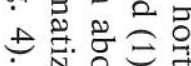
苛.

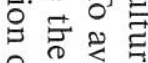
웅요. 루융 웅

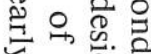
○完总 व.

虽寻完 至要零 드ㅇㅝㅠ 穴声总 的寻完 。 ङ $\stackrel{0}{0} \exists$ 


\section{References}

Al Maarri K, Arnaud Y, Miginiac E (1986) In vitro micropropagation of quince (Cydonia oblonga Mill.). Sci Hortic 28:315-321

Bajaj YPS (ed) (1986) Biotechnology in agriculture and forestry, vol 1. Trees I. Springer, Berlin Heidelberg New York Tokyo

Brossier J (1965) La sélection de porte-greffes du Poirier dans les populations naturelles de cognassier. I. Etude des populations naturelles de cognassier. Ann Amélior Plantes 15:263-326. II. Sélection de cognassiers porte-greffes du Poirier. Ann Amélior Plantes 15:373-404

Boxus PH, Quoirin M (1974) La culture de méristèmes apicaux de quelques espèces de Prunus. Bull Soc R Bot Belg 107:91-101

Childers NF (1976) Quince culture. In: Modern fruit science. Hortic Publ. Rutgers Univ, New Brunswick, NJ, pp 324-325

Collet GF (1985) Plant tissue culture laboratory at the Federal Research Institute of Changins. IAPTC Newslett 46:16-17

Druart Ph (1980) La micropropagation des nouveaux sujets porte-greffes nanifiants chez le Cerisier. Int Symp Cherry tree, Gembloux, Belgium

Dudits D, Nemeth G, Haydu Z (1975) Study of callus growth and organ formation in wheat (Triticum aestivum) tissue cultures. Can J Bot 53:957-963

Evreinoff VA (1948) Le cognassier. In: Les fruits à pépins. Collection la terre. Flammarion, Paris, pp $215-232$

Gautheret RJ (1959) La culture des tissues végétaux. Masson, Paris

Gur A, Samish RM, Lifshitz E (1968) The role of cyanogenic glycoside of the quince in the incompatibility between pear cultivars and quince rootstocks. Hortic Res 8:113-134

Hofinger M, Thorpe T, Bouchet M, Gaspar T (1980) Auxin-like activity of [benzo(b)selenienyl-3] acetic acid. Acta Physiol Plant 2:275-280

Jones OP, Hopgood ME, O'Farell D (1977) Propagation in vitro of M 26 apple rootstocks. J Hortic Sci 59:235-238

Lemoine J (1977) Susceptibility of several quince rootstocks to latent virus. Acta Hortic 75:133-138

Lemoine J, Michelesi JC (1984) Comportement des cognassiers AEM et Sydo greffés avec la variété Passe Crassane contaminés par diverses maladies de dégénérescence. Hortic Fr 164:3-7

Lepoivre $\mathrm{Ph}$ (1978) Table ronde: Multiplication in vitro d'espèces ligneuses, 6-8 Juin 1978. Service des relations publiques, Avenue de la Faculté d'Agronomie 22 - B-5800 Gembloux, Belgium

Michelesi JC, Brossier J, Flick JD (1973) Premières observations sur plusieurs variétés de cognassiers à fruits. Arboric Fruit 233:36-38

Moore R (1984) Ultrastructural aspects of graft incompatibility between pear and quince in vitro. Ann Bot (London) 53:447-451

Murashige T, Skoog F (1962) A revised medium for rapid growth and bioassays with tobacco tissue cultures. Physiol Plant 15:473-497

Nemeth G (1979) Benzyladenine-stimulated rooting in fruit tree rootstocks cultured in vitro. Z Pflanzenphysiol 95:388-396

Nemeth G (1986) Induction of rooting. In: Bajaj YPS (ed) Biotechnology in agriculture and forestry, vol 1. Trees I. Springer, Berlin Heidelberg New York, pp 49-64

Quoirin M, Lepoivre Ph, Boxus Ph (1977) Un premier bilan de 10 années de recherches sur les cultures de méristèmes et la multiplication in vitro des fruitiers ligneux. C R Rech 1976-1977. Stn Cult Fruit Maraîchères, Gembloux, pp 93-117

Rudenko IS (1978) Hybridization and polyploidy in fruit trees. In: Kichiniev, Chtiintsa (eds) 196 pp (in Russian) (Summary: Pl Breed Abstr 1980)

Walkey DG (1972) Production of apple plantlets from axillary-bud meristems. Can J Plant Sci 52:1085- 1087

Wilkins CP, Dodds JH (1982) Effect of various growth regulators on growth in vitro of cherry shoot tips. Plant Growth Regul 1:209-216

Williams RR, Taji AM, Bolton JA (1985) Specificity and interaction among auxins, light and pH on rooting of australian woody species in vitro. HortSci 20:1052-1053

Zimmerman RH, Fordham I (1985) Simplified method for rooting apple cultivars in vitro. J Am Soc Hortic Sci 110:34-38 